\title{
Positive periodic solutions for high-order differential equations with multiple delays in Banach spaces
}

Yue Liang ${ }^{1 *}$ and Hong Li ${ }^{1}$

"Correspondence:
liangyuegsau@163.com
'Center for Quantitative Biology,
College of Science, Gansu
Agricultural University, Lanzhou,
P.R. China

\begin{abstract}
This paper deals with the existence of positive $\omega$-periodic solutions for $n$ th-order ordinary differential equation with delays in Banach space $E$ of the form

$$
L_{n} u(t)=f\left(t, u\left(t-\tau_{1}\right), \ldots, u\left(t-\tau_{m}\right)\right), \quad t \in \mathbb{R}
$$

where $L_{n} u(t)=u^{(n)}(t)+\sum_{i=0}^{n-1} a_{i} u^{(i)}(t)$ is the $n$ th-order linear differential operator, $a_{i} \in \mathbb{R}$ $(i=0,1, \ldots, n-1)$ are constants, $f: \mathbb{R} \times E^{m} \rightarrow E$ is a continuous function which is $\omega$-periodic with respect to $t$, and $\tau_{i}>0(i=1,2, \ldots, m)$ are constants which denote the time delays. We first prove the existence of $\omega$-periodic solutions of the corresponding linear problem. Then the strong positivity estimation is established. Finally, two existence theorems of positive $\omega$-periodic solutions are proved. Our discussion is based on the theory of fixed point index in cones.
\end{abstract}

MSC: 34B18; 34C25

Keywords: $n$ th-order differential equation; Delays; Positive $\omega$-periodic solution; Existence; Fixed point index theory

\section{Introduction}

In recent years, the existence of periodic solutions for differential equations has been studied by many authors. But in some practice models, only positive periodic solutions are more important. For second-order differential equations without delay, the existence of positive periodic solutions has been discussed extensively, see $[1,5,10-12]$ and the references therein. In [2], Chu and Zhou considered the periodic solutions for the third-order differential equation

$$
u^{\prime \prime \prime}(t)+\rho^{3} u(t)=f(t, u(t)), \quad t \in[0,2 \pi]
$$

where $\rho \in\left(0, \frac{1}{\sqrt{3}}\right)$ is a constant and $f \in C([0,2 \pi] \times(0, \infty),[0,+\infty))$. By using the Krasnoselskii fixed point theorem in cones, they proved the existence of positive $2 \pi$-periodic

(c) The Author(s) 2020. This article is licensed under a Creative Commons Attribution 4.0 International License, which permits use, sharing, adaptation, distribution and reproduction in any medium or format, as long as you give appropriate credit to the original author(s) and the source, provide a link to the Creative Commons licence, and indicate if changes were made. The images or other third party material in this article are included in the article's Creative Commons licence, unless indicated otherwise in a credit line to the material. If material is not included in the article's Creative Commons licence and your intended use is not permitted by statutory regulation or exceeds the permitted use, you will need to obtain permission directly from the copyright holder. To view a copy of this licence, visit http://creativecommons.org/licenses/by/4.0/. 
solutions. In [4], Feng studied the third-order differential equation

$$
u^{\prime \prime \prime}(t)+\delta u^{\prime \prime}(t)+\varrho u^{\prime}(t)=f(t, u(t)), \quad t \in[0,2 \pi]
$$

where $\delta$ and $\varrho$ are positive constants. By utilizing the Guo-Krasnoselskii fixed point theorem in cones, he established some existence and multiplicity results of positive $2 \pi$ periodic solutions. For the more general case, in [7], Li proved some existence theorems of positive $2 \pi$-periodic solutions for the high-order differential equation

$$
L_{n} u(t)=f(t, u(t)), \quad t \in \mathbb{R},
$$

where $L_{n} u(t)=u^{(n)}(t)+\sum_{i=0}^{n-1} a_{i} u^{(i)}(t)$ is the $n$ th-order linear differential operator, $a_{i} \in \mathbb{R}$ $(i=0,1, \ldots, n-1)$ are constants. However, in these works, the authors did not consider the effect of the delay in the equation. Recently, Li [8] discussed the existence of positive $\omega$-periodic solutions of the second-order differential equation with delays of the form

$$
-u^{\prime \prime}(t)+a(t) u(t)=f\left(t, u\left(t-\tau_{1}\right), \ldots, u\left(t-\tau_{m}\right)\right), \quad t \in \mathbb{R},
$$

where $a \in C(\mathbb{R},(0, \infty))$ is an $\omega$-periodic function, $f: \mathbb{R} \times[0, \infty)^{m} \rightarrow[0, \infty)$ is a continuous function that is $\omega$-periodic with respect to $t$, and $\tau_{1}, \tau_{2}, \ldots, \tau_{m}$ are positive constants. The results obtained in [8] can deal with the case of second-order differential equations, but for high-order differential equations, for example,

$$
L_{n} u(t)=\frac{1}{2} u^{2}\left(t-\tau_{1}\right)+\frac{1}{4} u^{2}\left(t-\tau_{2}\right)+\frac{1}{8} u^{2}\left(t-\tau_{3}\right), \quad t \in \mathbb{R},
$$

the results of [8] are not valid.

Motivated by the papers mentioned above, we consider the existence of positive $\omega$ periodic solutions for the $n$ th-order nonlinear ordinary differential equations in Banach space $E$

$$
L_{n} u(t)=f\left(t, u\left(t-\tau_{1}\right), \ldots, u\left(t-\tau_{m}\right)\right), \quad t \in \mathbb{R},
$$

where $f: \mathbb{R} \times E^{m} \rightarrow E$ is a continuous function that is $\omega$-periodic with respect to $t$, and $\tau_{1}, \tau_{2}, \ldots, \tau_{m}$ are positive constants which denote the time delays.

The main features and crucial technique of the present paper are summarized as follows:

(i) In this paper, we discuss the effect of multiple delays in the high-order ordinary differential equation in abstract Banach spaces, which has seldom been studied before.

(ii) Since the integral operator $Q$ is not compact in abstract Banach spaces, the fixed theorems of completely continuous mapping are not valid for this problem. In order to overcome this difficulty, we provide a measure of non-compactness condition $(R 1)$ on nonlinear $\operatorname{term} f$, which is much weaker than some existing results. And we prove that the operator $Q$ is a condensing mapping, see Lemma 2.7.

(iii) By utilizing the perturbation method, we obtain the existence of positive $\omega$-periodic solution of the linear differential equation corresponding to Eq. (1.1). 
Then the strong positivity estimation of the operator $T$ is established by using the positivity of $G_{n}(t, s)$ and $T_{n}$, see Lemma 2.3 .

(iv) In our main results Theorem 3.1 and Theorem 3.2, we provide some order conditions on nonlinearity $f$ to guarantee the existence of positive $\omega$-periodic solutions of Eq. (1.1), which are much easier to verify in application.

The rest of this paper is organized as follows. In Sect. 2, we introduce some preliminaries and prove the existence of positive solutions of the corresponding linear problem. The main results of this paper are presented in Sect. 3. Some remarks are given to show the superiority of this work.

\section{Preliminaries}

Let $I=[0, \omega], C(I, \mathbb{R})$ be the Banach space of all continuous functions furnished with the norm $\|u\|_{C}=\max _{t \in I}|u(t)|$. For $\forall h \in C(I, \mathbb{R})$, we first consider the linear boundary value problem (LBVP)

$$
\left\{\begin{array}{l}
L_{n} u(t)=0, \quad t \in I, \\
u^{(i)}(0)=u^{(i)}(\omega), \quad i=0,1, \ldots, n-2, \\
u^{(n-1)}(0)-u^{(n-1)}(\omega)=1 .
\end{array}\right.
$$

Denote by $P_{n}(\lambda)$ the characteristic polynomial of $L_{n}$ :

$$
P_{n}(\lambda)=\lambda^{n}+a_{n-1} \lambda^{n-1}+\cdots+a_{0}
$$

Let $\mathcal{N}\left(P_{n}(\lambda)\right)=\left\{\lambda \in \mathbb{C}: P_{n}(\lambda)=0\right\}$, where $\mathbb{C}$ denotes the complex plane. By Lemma 3 of [7], we get the following lemma.

Lemma 2.1 Let $P_{n}(\lambda)$, the characteristic polynomial of $L_{n}$, satisfy

(P) $\mathcal{N}\left(P_{n}(\lambda)\right) \subset\left\{z \in \mathbb{C}:|\operatorname{Im} z|<\frac{\pi}{\omega}\right\}$.

Then, if $a_{0}>0, L B V P(2.1)$ has a unique solution $r_{n}(t)>0$ for any $t \in I$.

Let $E$ be a Banach space whose positive cone $K$ is normal. Denote by $C_{\omega}(\mathbb{R}, E)$ the Banach space of all $E$-valued $\omega$-periodic continuous functions on $\mathbb{R}$ endowed with the norm $\|u\|_{C}=\max _{t \in I}\|u(t)\|$. Let $K_{C}=C_{\omega}(\mathbb{R}, K)$ be the normal cone of $C_{\omega}(\mathbb{R}, E)$.

Definition 2.1 A function $u \in C_{\omega}^{n}(\mathbb{R}, E)$ is called a positive $\omega$-periodic solution of Eq. (1.1) if $u(t)>0$ for any $t \in \mathbb{R}$ and $u(t)$ satisfies Eq. (1.1).

Lemma 2.2 Let assumption $(P)$ hold and $a_{0}>0$. Then, for $\forall h \in C_{\omega}(\mathbb{R}, E)$, the linear equation

$$
L_{n} u(t)=h(t), \quad t \in \mathbb{R}
$$

has a unique $\omega$-periodic solution given by

$$
u(t)=\int_{t-\omega}^{t} G_{n}(t, s) h(s) d s:=\left(T_{n} h\right)(t), \quad t \in \mathbb{R},
$$


where

$$
G_{n}(t, s)= \begin{cases}r_{n}(t-s), & 0 \leq s \leq t \leq \omega \\ r_{n}(\omega+t-s), & 0 \leq t<s \leq \omega\end{cases}
$$

where $r_{n}(t) \in C^{\infty}(I, \mathbb{R})$ is the unique solution of $L B V P(2.1)$, and $T_{n}: C_{\omega}(\mathbb{R}, E) \rightarrow C_{\omega}(\mathbb{R}, E)$ is a positive and bounded linear operator, whose norm satisfies $\left\|T_{n}\right\|=\frac{1}{a_{0}}$.

Proof From Lemma 2.1, if assumption $(P)$ holds and $a_{0}>0$, LBVP(2.1) has a unique solution $r_{n}(t)>0$ for any $t \in I$. By Lemma 1 of [7], the linear periodic boundary value problem(LPBVP)

$$
\left\{\begin{array}{l}
L_{n} u(t)=h(t), \quad t \in I, \\
u^{(i)}(0)=u^{(i)}(\omega)
\end{array}\right.
$$

has a unique solution $u \in C^{n}(I, E)$, which is given by the expression

$$
u(t)=\int_{0}^{\omega} G_{n}(t, s) h(s) d s
$$

Since the $\omega$-extension of the solution of $\operatorname{LPBVP}(2.4)$ is the $\omega$-periodic solution of linear equation (2.2). Inversely, the $\omega$-periodic solution of linear equation (2.2) restricted to $[0, \omega]$ is the solution of $\operatorname{LPBVP}(2.4)$. Hence, for $\forall h \in C_{\omega}(\mathbb{R}, E)$, linear equation (2.2) has a unique $\omega$-periodic solution, which is given by (2.3).

Clearly, $T_{n}: C_{\omega}(\mathbb{R}, E) \rightarrow C_{\omega}^{n}(\mathbb{R}, E) \hookrightarrow C_{\omega}(\mathbb{R}, E)$ is a positive operator. It remains to prove that $T_{n}$ is bounded and $\left\|T_{n}\right\|=\frac{1}{a_{0}}$. On the one hand, for $\forall h \in C_{\omega}(\mathbb{R}, E)$, the inequality

$$
\left\|T_{n} h(t)\right\|=\left\|\int_{t-\omega}^{t} G_{n}(t, s) h(s) d s\right\| \leq \frac{1}{a_{0}}\|h\|_{C}
$$

implies $\left\|T_{n}\right\| \leq \frac{1}{a_{0}}$. This means that $T_{n}$ is bounded.

On the other hand, let $h_{0}(t) \equiv 1$ for all $t \in \mathbb{R}$. Then $h_{0} \in C_{\omega}(\mathbb{R}, E)$ and $\left\|h_{0}\right\|_{C}=1$. So,

$$
\begin{aligned}
\left\|T_{n} h_{0}(t)\right\| & =\left\|\int_{t-\omega}^{t} G_{n}(t, s) h_{0}(s) d s\right\| \\
& =\left\|\int_{0}^{\omega} G_{n}(t, s) d s\right\|\left\|h_{0}\right\|_{C} \\
& =\frac{1}{a_{0}}\left\|h_{0}\right\|_{C} .
\end{aligned}
$$

Therefore, $\left\|T_{n}\right\|=\frac{1}{a_{0}}$.

In order to prove the existence of positive $\omega$-periodic solutions of Eq. (1.1), for $\forall h \in$ $C_{\omega}(\mathbb{R}, E)$, we consider the linear differential equation with delay of the form

$$
L_{n} u(t)+\rho u(t-\tau)=h(t), \quad t \in \mathbb{R}
$$

where $\rho \geq 0$ is a constant. 
If $r_{n}(t)>0$ for $t \in I$, let $m_{n}=\min _{t \in I} r_{n}(t)$ and $M_{n}=\max _{t \in I} r_{n}(t)$. Then $0<m_{n} \leq r_{n}(t) \leq$ $M_{n}$. By Lemmas 2.1 and 2.2, we obtain the following lemma.

Lemma 2.3 Assume that $(P)$ holds and $0 \leq \rho<\gamma a_{0}$, where $\gamma=\frac{m_{n}}{M_{n}}$. Then, for $\forall h \in$ $C_{\omega}(\mathbb{R}, E)$, linear delayed differential equation (2.5) has a unique $\omega$-periodic solution $u:=$ $T h \in C_{\omega}(\mathbb{R}, E)$, and $T: C_{\omega}(\mathbb{R}, E) \rightarrow C_{\omega}(\mathbb{R}, E)$ is a linear bounded operator satisfying $\|T\| \leq$ $\frac{1}{a_{0}-\rho}$. If $h \in C_{\omega}(\mathbb{R}, K)$, then $T: C_{\omega}(\mathbb{R}, K) \rightarrow C_{\omega}(\mathbb{R}, K)$ is a positive linear bounded operator satisfying the strong positivity estimate

$$
(T h)(t) \geq \gamma(T h)(s), \quad \forall t, s \in \mathbb{R} .
$$

Proof By Lemma 2.2, the $\omega$-periodic solution of Eq. (2.5) is expressed by

$$
u(t)=\int_{t-\omega}^{t} G_{n}(t, s)[h(s)-\rho u(s-\tau)] d s, \quad t \in \mathbb{R} .
$$

Define an operator $B$ by

$$
B u(t)=\rho u(t-\tau), \quad t \in \mathbb{R} .
$$

Then $B: C_{\omega}(\mathbb{R}, E) \rightarrow C_{\omega}(\mathbb{R}, E)$ is a linear bounded operator with $\|B\| \leq \rho$. Hence, by (2.6) and (2.7), we have

$$
\left(I+T_{n} B\right) u(t)=T_{n} h(t), \quad t \in \mathbb{R} .
$$

Since $\left\|T_{n} B\right\| \leq\left\|T_{n}\right\|\|B\| \leq \frac{\rho}{a_{0}}<1$, by the perturbation theorem, $\left(I+T_{n} B\right)^{-1}$ exists and

$$
\left(I+T_{n} B\right)^{-1}=\sum_{i=0}^{\infty}(-1)^{i}\left(T_{n} B\right)^{i}=\sum_{i=0}^{\infty}\left(T_{n} B\right)^{2 i}\left(I-T_{n} B\right) .
$$

By direct calculation, we get

$$
\left\|\left(I+T_{n} B\right)^{-1}\right\| \leq \frac{a_{0}}{a_{0}-\rho} .
$$

Hence, by (2.8), we have

$$
u(t)=\left(I+T_{n} B\right)^{-1} T_{n} h(t):=T h(t), \quad t \in \mathbb{R},
$$

which is an $\omega$-periodic solution of (2.5). By (2.10) and (2.11), we have

$$
\begin{aligned}
\|T h(t)\| & =\left\|\left(I+T_{n} B\right)^{-1} T_{n} h(t)\right\| \\
& \leq\left\|\left(I+T_{n} B\right)^{-1}\right\|\left\|T_{n}\right\| \cdot\|h\|_{C} \\
& \leq \frac{1}{a_{0}-\rho}\|h\|_{C} .
\end{aligned}
$$

Therefore,

$$
\|T\| \leq \frac{1}{a_{0}-\rho}
$$


Furthermore, for $\forall h \in C_{\omega}(\mathbb{R}, K)$, we prove that $T: C_{\omega}(\mathbb{R}, K) \rightarrow C_{\omega}(\mathbb{R}, K)$ is a positive operator and $(T h)(t) \geq \gamma(T h)(s)$ for any $t, s \in \mathbb{R}$. By (2.9) and (2.11), we have

$$
T h(t)=\sum_{i=0}^{\infty}\left(T_{n} B\right)^{2 i}\left(I-T_{n} B\right) T_{n} h(t), \quad t \in \mathbb{R} .
$$

Since

$$
T_{n} h(t)=\int_{t-\omega}^{t} G_{n}(t, s) h(s) d s \geq m_{n} \int_{0}^{\omega} h(s) d s
$$

and

$$
T_{n} h(t) \leq M_{n} \int_{0}^{\omega} h(s) d s
$$

and

$$
\left(T_{n} B\right) T_{n} h(t) \leq \frac{\rho M_{n}}{a_{0}} \int_{0}^{\omega} h(s) d s
$$

we get

$$
\begin{aligned}
\left(I-T_{n} B\right) T_{n} h(t) & =T_{n} h(t)-\left(T_{n} B\right) T_{n} h(t) \\
& \geq m_{n} \int_{0}^{\omega} h(s) d s-\frac{\rho M_{n}}{a_{0}} \int_{0}^{\omega} h(s) d s \\
& =\left(m_{n}-\frac{\rho M_{n}}{a_{0}}\right) \int_{0}^{\omega} h(s) d s .
\end{aligned}
$$

Since $h \in C_{\omega}(\mathbb{R}, K), h(t) \not \equiv 0$ for all $t \in \mathbb{R}$. There exist $[c, d] \subset I$ and $\varepsilon>0$ such that

$$
h(t)>\varepsilon, \quad t \in[c, d]
$$

from which we get $\int_{0}^{\omega} h(s) d s \geq \int_{c}^{d} h(s) d s>\varepsilon(d-c)>0$. Due to $\rho<\gamma a_{0}$, we get

$$
\begin{aligned}
\left(I-T_{n} B\right) T_{n} h(t) & \geq\left(m_{n}-\frac{\rho M_{n}}{a_{0}}\right) \int_{0}^{\omega} h(s) d s \\
& \geq \frac{a_{0} m_{n}-\rho M_{n}}{a_{0}} \varepsilon(d-c) \\
& >0 .
\end{aligned}
$$

Therefore, $T: C_{\omega}(\mathbb{R}, K) \rightarrow C_{\omega}(\mathbb{R}, K)$ is a positive operator. Moreover, for any $h \in C_{\omega}(\mathbb{R}, K)$, by (2.11), we have

$$
\left(I+T_{n} B\right)(T h)(t)=T_{n} h(t)=\int_{t-\omega}^{t} G_{n}(t, s) h(s) d s, \quad t \in \mathbb{R} .
$$

So, we have

$$
\left(I+T_{n} B\right)(T h)(t) \geq m_{n} \int_{0}^{\omega} h(s) d s
$$


and

$$
\int_{0}^{\omega} h(s) d s \geq \frac{1}{M_{n}}\left(I+T_{n} B\right)(T h)(t) .
$$

Consequently, for any $t, s \in \mathbb{R}$, we have

$$
\left(I+T_{n} B\right)(T h)(t) \geq \gamma\left(I+T_{n} B\right)(T h)(s) .
$$

Hence, $(T h)(t) \geq \gamma(T h)(s)$ for any $t, s \in \mathbb{R}$.

Let $E$ be a separable Banach space. Denote by $\beta_{E}(\cdot)$ and $\beta_{C}(\cdot)$ the Hausdorff measure of non-compactness(MNC) of the bounded set in $E$ and $C_{\omega}(\mathbb{R}, E)$, respectively. Let $D \subset$ $C_{\omega}(\mathbb{R}, E)$ be bounded, set $D(t)=\{u(t): u \in D\} \subset E$ for $t \in \mathbb{R}$. Then $\beta_{E}(D(t)) \leq \beta_{C}(D)$. The following lemmas for the MNC are cited from $[3,6,13]$.

Lemma 2.4 Let $D \subset C(I, E)$ be a bounded and equicontinuous subset. Then $\beta_{E}(D(t))$ is continuous on I and

$$
\beta_{C}(D)=\max _{t \in I} \beta_{E}(D(t))=\beta_{E}(D(I))
$$

where $D(I):=\{u(t): u \in D, t \in I\}$.

Lemma 2.5 Let $D \subset E$ be bounded. Then there exists a countable subset $D_{0} \subset D$ such that

$$
\beta_{E}(D) \leq 2 \beta_{E}\left(D_{0}\right)
$$

Lemma 2.6 Let $D=\left\{u_{n}\right\} \subset C(I, E)$ be a bounded and countable subset. Then $\beta_{E}(D(t))$ is Lebesgue integrable on I and

$$
\beta_{E}\left(\left\{\int_{I} u_{n}(t) d t\right\}\right) \leq 2 \int_{I} \beta_{E}(D(t)) d t .
$$

Now, we consider the existence of positive $\omega$-periodic solutions for the high-order differential equation with delays of the form (1.1). By Lemma 2.3, we define an operator $Q: C_{\omega}(\mathbb{R}, E) \rightarrow C_{\omega}(\mathbb{R}, E)$ by

$$
\begin{aligned}
(Q u)(t)= & \left(I+T_{n} B\right)^{-1} \\
& \times \int_{t-\omega}^{t} G_{n}(t, s)\left[f\left(s, u\left(s-\tau_{1}\right), \ldots, u\left(s-\tau_{m}\right)\right)+\rho u\left(s-\tau_{1}\right)\right] d s, \quad t \in \mathbb{R} .
\end{aligned}
$$

By the continuity of $f$, the operator $Q: C_{\omega}(\mathbb{R}, E) \longrightarrow C_{\omega}(\mathbb{R}, E)$ is continuous. The positive $\omega$-periodic solution of the high-order differential equation (1.1) is equivalent to the positive fixed point of $Q$. It is noted that the integral operator $Q$ is not compact in an abstract Banach space. In order to employ the topological degree theory of condensing mapping, it demands that the nonlinear term $f$ satisfies some MNC conditions. Thus, we make the following assumption. 
(R1) For $\forall r>0, f \in C\left(\mathbb{R} \times K_{r}^{m}, E\right)$ is bounded and

$$
\beta_{E}\left(f\left(t, D_{1}, D_{2}, \ldots, D_{m}\right)+\rho D_{1}\right) \leq \sum_{i=1}^{m} M_{i} \beta_{E}\left(D_{i}\right), \quad t \in \mathbb{R}
$$

where $K_{r}=\{u \in K:\|u\| \leq r\}, D_{i} \subset K_{r}(i=1,2, \ldots, m)$ are arbitrarily countable subsets, $M_{i}(i=1,2, \ldots, m)$ are positive constants satisfying

$$
\sum_{i=1}^{m} M_{i}<\frac{a_{0}-\rho}{4}
$$

Lemma 2.7 Suppose that condition $(R 1)$ holds. Then $Q: C_{\omega}\left(\mathbb{R}, K_{r}\right) \rightarrow C_{\omega}(\mathbb{R}, K)$ is a condensing mapping.

Proof For any $r>0$, let $K_{r, C}=\left\{u \in C_{\omega}(\mathbb{R}, K): u(t) \in K_{r}, t \in \mathbb{R}\right\}$. Since $f\left(\mathbb{R} \times K_{r}^{m}, E\right)$ is bounded, there exists a constant $\bar{M}>0$ such that

$$
\left\|f\left(t, x_{1}, \ldots, x_{m}\right)\right\| \leq \bar{M}
$$

for any $t \in \mathbb{R}$ and $x_{i} \in K_{r}, i=1,2, \ldots, m$. Hence, for any $u \in K_{r, C}$, by (2.15), we have

$$
\begin{aligned}
\|(Q u)(t)\| & =\left\|\left(I+T_{n} B\right)^{-1} \int_{t-\omega}^{t} G_{n}(t, s)\left[f\left(s, u\left(s-\tau_{1}\right), \ldots, u\left(s-\tau_{m}\right)\right)+\rho u\left(s-\tau_{1}\right)\right] d s\right\| \\
& \leq \frac{a_{0}}{a_{0}-\rho} \int_{t-\omega}^{t} G_{n}(t, s)\left\|f\left(s, u\left(s-\tau_{1}\right), \ldots, u\left(s-\tau_{m}\right)\right)+\rho u\left(s-\tau_{1}\right)\right\| d s \\
& \leq \frac{a_{0}}{a_{0}-\rho} \int_{0}^{\omega} G_{n}(t, s)[\bar{M}+\rho r] d s \\
& =\frac{\bar{M}+\rho r}{a_{0}-\rho} .
\end{aligned}
$$

Then $Q\left(K_{r, C}\right)$ is bounded. Clearly, $Q\left(K_{r, C}\right)$ is equicontinuous. Hence, by Lemmas 2.4 and 2.5, there exists a countable subset $D_{\ell}=\left\{u_{\ell}\right\}_{\ell=1}^{\infty} \subset K_{r, C}$ such that

$$
\beta_{C}\left(Q\left(K_{r, C}\right)\right) \leq 2 \beta_{C}\left(Q\left(D_{\ell}\right)\right)=2 \max _{t \in J} \beta_{E}\left(Q\left(D_{\ell}\right)(t)\right)
$$

By assumption $(R 1)$ and Lemma 2.6, we have

$$
\begin{aligned}
& \beta_{E}\left(Q\left(D_{\ell}\right)(t)\right) \\
& =\beta_{E}\left(\left\{\left(I+T_{n} B\right)^{-1} \int_{t-\omega}^{t} G_{n}(t, s)\left[f\left(s, u_{\ell}\left(s-\tau_{1}\right), \ldots, u_{\ell}\left(s-\tau_{m}\right)\right)+\rho u_{\ell}\left(s-\tau_{1}\right)\right] d s\right\}\right) \\
& \leq \frac{2 a_{0}}{a_{0}-\rho} \int_{t-\omega}^{t} G_{n}(t, s) \beta_{E}\left(\left\{f\left(s, u_{\ell}\left(s-\tau_{1}\right), \ldots, u_{\ell}\left(s-\tau_{m}\right)\right)+\rho u_{\ell}\left(s-\tau_{1}\right)\right\}\right) d s \\
& \leq \frac{2 a_{0}}{a_{0}-\rho} \sum_{i=1}^{m} M_{i} \int_{t-\omega}^{t} G_{n}(t, s) \beta_{E}\left(\left\{u_{\ell}\left(s-\tau_{i}\right)\right\}\right) d s
\end{aligned}
$$




$$
\begin{aligned}
& \leq \frac{2 a_{0}}{a_{0}-\rho} \sum_{i=1}^{m} M_{i} \int_{0}^{\omega} G_{n}(t, s) d s \beta_{C}\left(D_{\ell}\right) \\
& \leq \frac{2 \sum_{i=1}^{m} M_{i}}{a_{0}-\rho} \beta_{C}\left(K_{r, C}\right) .
\end{aligned}
$$

Consequently, we have

$$
\beta_{C}\left(Q\left(K_{r, C}\right)\right) \leq 2 \max _{t \in J} \beta_{E}\left(Q\left(D_{\ell}\right)(t)\right) \leq \frac{4 \sum_{i=1}^{m} M_{i}}{a_{0}-\rho} \beta_{C}\left(K_{r, C}\right)
$$

Hence, $Q: C_{\omega}\left(\mathbb{R}, K_{r}\right) \longrightarrow C_{\omega}(\mathbb{R}, K)$ is a condensing mapping due to (2.16).

Remark 1 In Lemma 2.7, if the nonlinearity $f$ satisfies linear growth condition, for example, $f$ satisfies the following condition:

(R2) There exist constants $\bar{C}_{i}>0(i=1,2, \ldots, m)$ and $b>0$ such that

$$
f\left(t, x_{1}, \ldots, x_{m}\right) \leq \sum_{i=1}^{m} \bar{C}_{i} x_{i}+b
$$

for any $t \in \mathbb{R}$ and $x_{i} \in K, i=1,2, \ldots, m$, then (2.17) holds for $x_{i} \in K_{r}, i=1,2, \ldots, m$, with $\bar{M}=\sum_{i=1}^{m} \bar{C}_{i} r+b$.

Define a cone $\Xi$ in $C_{\omega}(\mathbb{R}, K)$ by

$$
\Xi=\left\{u \in C_{\omega}(\mathbb{R}, K): u(t) \geq \gamma u(s), \forall t, s \in \mathbb{R}\right\},
$$

where $\gamma=\frac{m_{n}}{M_{n}}$. Then we can obtain the following lemma.

Lemma 2.8 $Q\left(C_{\omega}(\mathbb{R}, K)\right) \subset \Xi$.

Proof For any $t, s \in \mathbb{R}$ and $u \in C_{\omega}(\mathbb{R}, K)$, by (2.15), we have

$$
\begin{aligned}
\left(I+T_{n} B\right)(Q u)(t) & =\int_{t-\omega}^{t} G_{n}(t, \theta)\left[f\left(\theta, u\left(\theta-\tau_{1}\right), \ldots, u\left(\theta-\tau_{m}\right)\right)+\rho u\left(\theta-\tau_{1}\right)\right] d \theta \\
& \geq m_{n} \int_{0}^{\omega} f\left(\theta, u\left(\theta-\tau_{1}\right), \ldots, u\left(\theta-\tau_{m}\right)\right)+\rho u\left(\theta-\tau_{1}\right) d \theta
\end{aligned}
$$

and

$$
\begin{aligned}
\left(I+T_{n} B\right)(Q u)(s) & =\int_{s-\omega}^{s} G_{n}(s, \theta)\left[f\left(\theta, u\left(\theta-\tau_{1}\right), \ldots, u\left(\theta-\tau_{m}\right)\right)+\rho u\left(\theta-\tau_{1}\right)\right] d \theta \\
& \leq M_{n} \int_{0}^{\omega} f\left(\theta, u\left(\theta-\tau_{1}\right), \ldots, u\left(\theta-\tau_{m}\right)\right)+\rho u\left(\theta-\tau_{1}\right) d \theta .
\end{aligned}
$$

It follows from the above two inequalities that

$$
\left(I+T_{n} B\right)(Q u)(t) \geq \gamma\left(I+T_{n} B\right)(Q u)(s), \quad \forall t, s \in \mathbb{R} .
$$

Hence $Q\left(C_{\omega}(\mathbb{R}, K)\right) \subset \Xi$. 
Let $E$ be a Banach space and $D \subset E$ be a closed convex cone in $E$. Assume that $\Omega$ is a bounded open subset of $E$ with boundary $\partial \Omega$ and $D \cap \Omega \neq \emptyset$, and $Q: D \cap \bar{\Omega} \rightarrow D$ is a condensing mapping. If $Q u \neq u$ for any $u \in D \cap \partial \Omega$, the fixed point index $i(Q, D \cap \Omega, D)$ is well defined. If $i(Q, D \cap \Omega, D) \neq 0$, then $Q$ has a fixed point in $D \cap \Omega$. In the proof of the main results, the following two lemmas are useful.

Lemma 2.9 ([9]) Let $\Omega$ be a bounded open subset of $E$ with $\theta \in \Omega$ and $Q: D \cap \bar{\Omega} \rightarrow D$ be a condensing mapping. If

$$
\lambda Q u \neq u, \quad \forall u \in D \cap \partial \Omega, 0<\lambda \leq 1,
$$

then $i(Q, D \cap \Omega, D)=1$.

Lemma 2.10 ([9]) Let $\Omega$ be a bounded open subset of $E$ and $Q: D \cap \bar{\Omega} \rightarrow D$ be a condensing mapping. If there exists $e \in D \backslash\{\theta\}$ such that

$$
u-Q u \neq \mu e, \quad \forall u \in D \cap \partial \Omega, \mu \geq 0,
$$

then $i(Q, D \cap \Omega, D)=0$.

\section{Existence of positive $\omega$-periodic solutions}

Let $E$ be a separable Banach space and $K \subset E$ be a positive cone of $E$. For any positive constants $R$ and $r$, let

$$
\Omega_{R}=\left\{u \in C_{\omega}(\mathbb{R}, K):\|u\|_{C}<R\right\}, \quad \Omega_{r}=\left\{u \in C_{\omega}(\mathbb{R}, K):\|u\|_{C}<r\right\} .
$$

Then $\partial \Omega_{R}=\left\{u \in C_{\omega}(\mathbb{R}, K):\|u\|_{C}=R\right\}$ and $\partial \Omega_{r}=\left\{u \in C_{\omega}(\mathbb{R}, K):\|u\|_{C}=r\right\}$. Define an operator $Q: C_{\omega}(\mathbb{R}, K) \rightarrow C_{\omega}(\mathbb{R}, K)$ by $(2.15)$. Then, by Lemmas 2.7 and 2.8, $Q: C_{\omega}(\mathbb{R}, K) \rightarrow$ $C_{\omega}(\mathbb{R}, K)$ is a condensing mapping when assumption $(R 1)$ holds. We will prove that the operator $Q$ has at least one fixed point in $\Omega_{r, R}:=\Omega_{R} \backslash \bar{\Omega}_{r}$, which is the positive $\omega$-periodic solution of Eq. (1.1).

Theorem 3.1 Suppose that $(P)$ holds and $0 \leq \rho<\gamma a_{0}$. Let $f \in C\left(\mathbb{R} \times K^{m}, K\right)$ satisfy assumption (R1). Then Eq. (1.1) has at least one positive $\omega$-periodic solution if the following conditions hold:

(H1) There exist positive constants $c_{1}, \ldots, c_{m}$ satisfying $\sum_{i=1}^{m} c_{i}<\gamma^{2} a_{0}$ and $\delta>0$ such that

$$
f\left(t, x_{1}, \ldots, x_{m}\right) \leq \sum_{i=1}^{m} c_{i} x_{i}
$$

for any $t \in \mathbb{R}$ and $x_{i} \in K$ with $\left\|x_{i}\right\|<\delta, i=1,2, \ldots, m$.

(H2) There exist positive constants $d_{1}, \ldots, d_{m}$ satisfying $\sum_{i=1}^{m} d_{i}>a_{0}$ and $h_{0} \in C_{\omega}(\mathbb{R}, K)$ such that

$$
f\left(t, x_{1}, \ldots, x_{m}\right) \geq \sum_{i=1}^{m} d_{i} x_{i}-h_{0}(t)
$$

for any $t \in \mathbb{R}$ and $x_{i} \in \Xi, i=1,2, \ldots, m$. 
Proof Let $\Xi$ be the closed convex cone of $C_{\omega}(\mathbb{R}, K)$ defined by (2.20). Define an operator $Q: C_{\omega}(\mathbb{R}, K) \rightarrow C_{\omega}(\mathbb{R}, K)$ by (2.15). We show that $Q$ has a fixed point in $\Xi \cap \Omega_{r, R}$ for $r>0$ small enough and $R>0$ sufficiently large.

Let $r \in(0, \delta)$, where $\delta$ is the positive constant in assumption (H1). We prove that $Q$ satisfies the conditions of Lemma 2.9 in $\Xi \cap \Omega_{r}$, namely,

$$
\lambda Q u \neq u, \quad \forall u \in \Xi \cap \partial \Omega_{r}, 0<\lambda \leq 1 .
$$

In fact, if there exist $u_{0} \in \Xi \cap \partial \Omega_{r}$ and $0<\lambda_{0} \leq 1$ such that

$$
\lambda_{0} Q u_{0}=u_{0}
$$

then by the definition of $Q$ and Lemma 2.3, $u_{0}$ satisfies the delayed differential equation

$$
L_{n} u_{0}(t)+\rho u_{0}\left(t-\tau_{1}\right)=\lambda_{0} f\left(t, u_{0}\left(t-\tau_{1}\right), \ldots, u_{0}\left(t-\tau_{m}\right)\right)+\lambda_{0} \rho u_{0}\left(t-\tau_{1}\right), \quad t \in \mathbb{R},
$$

i.e.,

$$
L_{n} u_{0}(t) \leq f\left(t, u_{0}\left(t-\tau_{1}\right), \ldots, u_{0}\left(t-\tau_{m}\right)\right), \quad t \in \mathbb{R}
$$

Since $u_{0} \in \partial \Omega_{r}$, by the definition of $\Omega_{r}$, we have

$$
0 \leq\left\|u_{0}\left(t-\tau_{i}\right)\right\| \leq\left\|u_{0}\right\|_{C}=r<\delta, \quad i=1,2, \ldots, m, t \in \mathbb{R}
$$

It follows from $(H 1)$ that

$$
f\left(t, u_{0}\left(t-\tau_{1}\right), \ldots, u_{0}\left(t-\tau_{m}\right)\right) \leq \sum_{i=1}^{m} c_{i} u_{0}\left(t-\tau_{i}\right), \quad t \in \mathbb{R} .
$$

Hence, by (3.2), we have

$$
L_{n} u_{0}(t) \leq \sum_{i=1}^{m} c_{i} u_{0}\left(t-\tau_{i}\right), \quad t \in \mathbb{R} .
$$

Integrating both sides of this inequality from 0 to $\omega$ and using the periodicity of $u_{0}$, we have

$$
a_{0} \int_{0}^{\omega} u_{0}(\nu) d \nu \leq \sum_{i=1}^{m} c_{i} \int_{0}^{\omega} u_{0}\left(\nu-\tau_{i}\right) d \nu=\sum_{i=1}^{m} c_{i} \int_{0}^{\omega} u_{0}(\theta) d \theta .
$$

By the definition of cone $\Xi$, we have

$$
\int_{0}^{\omega} u_{0}(v) d v \geq \int_{0}^{\omega} \gamma u_{0}(s) d v \geq \gamma \omega u_{0}(s), \quad \forall v, s \in \mathbb{R}
$$

and

$$
\int_{0}^{\omega} u_{0}(v) d v \leq \frac{1}{\gamma} \omega u_{0}(t), \quad \forall v, t \in \mathbb{R} .
$$


By the arbitrariness of $t, s \in \mathbb{R}$ in (3.3) and (3.4), choosing $t=s$, we get

$$
a_{0} \gamma \omega u_{0}(s) \leq a_{0} \int_{0}^{\omega} u_{0}(v) d v \leq \sum_{i=1}^{m} c_{i} \int_{0}^{\omega} u_{0}(v) d v \leq \frac{1}{\gamma} \omega u_{0}(s) \sum_{i=1}^{m} c_{i}
$$

Consequently,

$$
\left(a_{0} \gamma \omega-\frac{1}{\gamma} \omega \sum_{i=1}^{m} c_{i}\right) u_{0}(s) \leq 0
$$

Since $\sum_{i=1}^{m} c_{i} \leq a_{0} \gamma^{2}$, it follows that $u_{0}(s) \leq 0$ for $s \in \mathbb{R}$, which is a contradiction to $u_{0} \in$ $\partial \Omega_{r}$. Hence, for any $u \in \Xi \cap \partial \Omega_{r}$ and $0<\lambda \leq 1$, we have

$\lambda Q u \neq u$.

By Lemma 2.9, we have

$$
i\left(Q, \Xi \cap \Omega_{r}, \Xi\right)=1 \text {. }
$$

Let $e \in C(\mathbb{R}, K)$ with $e(t) \equiv 1$ for any $t \in \mathbb{R}$. Then $e \in \Xi \backslash\{\theta\}$. We show that $Q$ satisfies the conditions of Lemma 2.10 in $\Xi \cap \partial \Omega_{R}$, that is,

$$
u-Q u \neq \mu e, \quad \forall u \in \Xi \cap \partial \Omega_{R}, \mu \geq 0
$$

for $R>0$ sufficiently large. In fact, if there exist $u_{1} \in \Xi \cap \partial \Omega_{R}$ and $\mu_{1} \geq 0$ such that

$$
u_{1}-\mu_{1} e=Q u_{1}
$$

Then, by the definition of $Q$ and Lemma $2.3, u_{1}$ satisfies the delayed differential equation

$$
L_{n} u_{1}(t)-\mu_{1} a_{0}=f\left(t, u_{1}\left(t-\tau_{1}\right), \ldots, u_{1}\left(t-\tau_{m}\right)\right), \quad t \in \mathbb{R}
$$

Since $u_{1} \in \Xi \cap \partial \Omega_{R}$, by condition $(H 2)$, we have

$$
L_{n} u_{1}(t) \geq \sum_{i=1}^{m} d_{i} u_{1}\left(t-\tau_{i}\right)-h_{0}(t)
$$

Integrating both sides of this inequality from 0 to $\omega$ and using the periodicity of $u_{1}$, we have

$$
\begin{aligned}
a_{0} \int_{0}^{\omega} u_{1}(v) d v & \geq \sum_{i=1}^{m} d_{i} \int_{0}^{\omega} u_{1}\left(v-\tau_{i}\right) d v-\int_{0}^{\omega} h_{0}(v) d v \\
& =\sum_{i=1}^{m} d_{i} \int_{0}^{\omega} u_{1}(v) d v-\int_{0}^{\omega} h_{0}(v) d v
\end{aligned}
$$


This implies

$$
\left(\sum_{i=1}^{m} d_{i}-a_{0}\right) \int_{0}^{\omega} u_{1}(v) d v \leq \int_{0}^{\omega} h_{0}(v) d v \leq \omega\left\|h_{0}\right\|_{C} .
$$

Since $u_{1} \in \Xi \cap \partial \Omega_{R}$, by the definition of $\Xi$, we have

$$
u_{1}(v) \geq \gamma u_{1}(s), \quad \forall v, s \in \mathbb{R}
$$

Hence, by (3.7) and $\sum_{i=1}^{m} d_{i}>a_{0}$, we have

$$
\left(\sum_{i=1}^{m} d_{i}-a_{0}\right) \omega \gamma u_{1}(s) \leq \omega\left\|h_{0}\right\|_{C}
$$

So,

$$
u_{1}(s) \leq \frac{\left\|h_{0}\right\|_{C}}{\gamma\left(\sum_{i=1}^{m} d_{i}-a_{0}\right)} \triangleq R^{*}
$$

Let $R>\max \left\{R^{*}, \delta\right\}$. Then (3.6) is satisfied. By Lemma 2.10, we have

$$
i\left(Q, \Xi \cap \Omega_{R}, \Xi\right)=0 .
$$

Combining (3.5) with (3.8), we have

$$
i\left(Q, \Xi \cap \Omega_{r, R}, \Xi\right)=i\left(Q, \Xi \cap \Omega_{R}, \Xi\right)-i\left(Q, \Xi \cap \Omega_{r}, \Xi\right)=-1 \neq 0 .
$$

Hence, $Q$ has at least one fixed point in $\Xi \cap \Omega_{r, R}$, which is the positive $\omega$-periodic solution of Eq. (1.1).

Remark 2 If we choose

$$
f\left(t, u\left(t-\tau_{1}\right), \ldots, u\left(t-\tau_{m}\right)\right)=\sum_{i=1}^{m} \frac{1}{i} u^{2}\left(t-\tau_{i}\right), \quad \forall u \in K_{C},
$$

we can prove that $(H 1)$ and $(H 2)$ hold. Hence, conditions $(H 1)$ and $(H 2)$ allow $f\left(t, x_{1}, \ldots, x_{m}\right)$ to be superlinear growth on $x_{1}, \ldots, x_{m}$.

Theorem 3.2 Suppose that assumption $(P)$ holds and $0 \leq \rho<\gamma a_{0}$. Let $f \in C\left(\mathbb{R} \times K^{m}, K\right)$ satisfy (R1). Then Eq. (1.1) has at least one positive $\omega$-periodic solution if the following conditions hold:

(H3) There exist positive constants $d_{1}, d_{2}, \ldots, d_{m}$ satisfying $\sum_{i=1}^{m} d_{i}>a_{0}$ and $\delta>0$ such that

$$
f\left(t, x_{1}, \ldots, x_{m}\right) \geq \sum_{i=1}^{m} d_{i} x_{i}
$$

for any $t \in \mathbb{R}$ and $x_{i} \in K$ with $\left\|x_{i}\right\|<\delta, i=1,2, \ldots, m$. 
(H4) There exist positive constants $c_{1}, c_{2}, \ldots, c_{m}$ satisfying $\sum_{i=1}^{m} c_{i}<a_{0}$ and $h_{1} \in C_{\omega}(\mathbb{R}, K)$ such that

$$
f\left(t, x_{1}, \ldots, x_{m}\right) \leq \sum_{i=1}^{m} c_{i} x_{i}+h_{1}(t)
$$

for any $t \in \mathbb{R}$ and $x_{i} \in \Xi, i=1,2, \ldots, m$.

Proof For any $0<r<R<+\infty$, choose $\Xi, \Omega_{r}, \Omega_{R}$, and $\Omega_{r, R}$ as in the proof of Theorem 3.1. Define an operator $Q$ by (2.15), then by $(R 1), Q: C_{\omega}(\mathbb{R}, K) \rightarrow C_{\omega}(\mathbb{R}, K)$ is a condensing mapping. We will show that the operator $Q$ has at least one fixed point in $\Xi \cap \Omega_{r, R}$.

Let $r \in(0, \delta)$. On the one hand, we prove that $Q$ satisfies the conditions of Lemma 2.10 in $\Xi \cap \partial \Omega_{r}$. Choose $e(t) \equiv 1$ for any $t \in \mathbb{R}$, then $e \in \Xi \backslash\{\theta\}$. For any $u \in \Xi \cap \partial \Omega_{r}$ and $\mu \geq 0$, we will show that

$$
u-\mu e \neq Q u \text {. }
$$

In fact, if there exist $u_{0} \in \Xi \cap \partial \Omega_{r}$ and $\mu_{0} \geq 0$ such that

$$
u_{0}-\mu_{0} e=Q u_{0}
$$

then $u_{0}$ satisfies the delayed differential equation

$$
L_{n} u_{0}(t)+\rho u_{0}\left(t-\tau_{1}\right)-\mu_{0} a_{0}=f\left(t, u_{0}\left(t-\tau_{1}\right), \ldots, u_{0}\left(t-\tau_{m}\right)\right)+\rho u_{0}\left(t-\tau_{1}\right), \quad t \in \mathbb{R}
$$

namely,

$$
L_{n} u_{0}(t)-\mu_{0} a_{0}=f\left(t, u_{0}\left(t-\tau_{1}\right), \ldots, u_{0}\left(t-\tau_{m}\right)\right), \quad t \in \mathbb{R}
$$

Since $u_{0} \in \Xi \cap \partial \Omega_{r}$, we have

$$
0 \leq\left\|u_{0}\left(t-\tau_{i}\right)\right\| \leq\left\|u_{0}\right\|_{C}=r<\delta
$$

So, by condition $(H 3)$, we have

$$
f\left(t, u_{0}\left(t-\tau_{1}\right), \ldots, u_{0}\left(t-\tau_{m}\right)\right) \geq \sum_{i=1}^{m} d_{i} u_{0}\left(t-\tau_{i}\right)
$$

for any $t \in \mathbb{R}$. Hence

$$
L_{n} u_{0}(t)-\mu_{0} a_{0} \geq \sum_{i=1}^{m} d_{i} u_{0}\left(t-\tau_{i}\right)
$$

Integrating both sides of this inequality from 0 to $\omega$, we have

$$
a_{0} \int_{0}^{\omega} u_{0}(v) d v-\mu_{0} a_{0} \omega \geq \sum_{i=1}^{m} d_{i} \int_{0}^{\omega} u_{0}\left(v-\tau_{i}\right) d v=\sum_{i=1}^{m} d_{i} \int_{0}^{\omega} u_{0}(v) d v
$$


So, we have

$$
\left(\sum_{i=1}^{m} d_{i}-a_{0}\right) \int_{0}^{\omega} u_{0}(v) d v \leq-\mu_{0} a_{0} \omega .
$$

Furthermore, we get

$$
\left(\sum_{i=1}^{m} d_{i}-a_{0}\right) \gamma \omega u_{0}(s) \leq-\mu_{0} a_{0} \omega
$$

In view of $\sum_{i=1}^{m} d_{i}>a_{0}, \gamma>0$, and $\omega>0$, we obtain that $u_{0}(s) \leq-\mu_{0} a_{0} \omega<0$ for any $s \in \mathbb{R}$, which contradicts $u_{0} \in \partial \Omega_{r}$. Hence, all the conditions of Lemma 2.10 hold. By Lemma 2.10, we have

$$
i\left(Q, \Xi \cap \partial \Omega_{r}, \Xi\right)=1 \text {. }
$$

On the other hand, we show that the conditions of Lemma 2.9 are satisfied when $R$ is large enough. That is, for any $u \in \Xi \cap \partial \Omega_{R}$ and $0<\lambda \leq 1$ such that

$$
u \neq \lambda Q u .
$$

In fact, if there exist $u_{1} \in \Xi \cap \partial \Omega_{R}$ and $0<\lambda_{1} \leq 1$ satisfying

$$
u_{1}=\lambda_{1} Q u_{1},
$$

then by the definition of $Q$, we have

$$
L_{n} u_{1}(t)+\rho u_{1}\left(t-\tau_{1}\right)=\lambda_{1} f\left(t, u_{1}\left(t-\tau_{1}\right), \ldots, u_{1}\left(t-\tau_{m}\right)\right)+\lambda_{1} \rho u_{1}\left(t-\tau_{1}\right), \quad t \in \mathbb{R} .
$$

Hence, we get

$$
L_{n} u_{1}(t) \leq f\left(t, u_{1}\left(t-\tau_{1}\right), \ldots, u_{1}\left(t-\tau_{m}\right)\right), \quad t \in \mathbb{R} .
$$

By virtue of $u_{1} \in \Xi \cap \partial \Omega_{R}$ and (H4), we have

$$
f\left(t, u_{1}\left(t-\tau_{1}\right), \ldots, u_{1}\left(t-\tau_{m}\right)\right) \leq \sum_{i=1}^{m} c_{i} u_{1}\left(t-\tau_{i}\right)+h_{1}(t), \quad t \in \mathbb{R} .
$$

Thus,

$$
L_{n} u_{1}(t) \leq \sum_{i=1}^{m} c_{i} u_{1}\left(t-\tau_{i}\right)+h_{1}(t), \quad t \in \mathbb{R}
$$

Integrating both sides of this inequality from 0 to $\omega$, we have

$$
a_{0} \int_{0}^{\omega} u_{1}(v) d v \leq \sum_{i=1}^{m} c_{i} \int_{0}^{\omega} u_{1}(v) d v+\int_{0}^{\omega} h_{1}(v) d v
$$


namely,

$$
\left(a_{0}-\sum_{i=1}^{m} c_{i}\right) \int_{0}^{\omega} u_{1}(v) d v \leq \int_{0}^{\omega} h_{1}(v) d v \leq \omega\left\|h_{1}\right\|_{C} .
$$

Since $u_{1} \in \Xi$, it follows that

$$
u_{1}(v) \geq \gamma u_{1}(s), \quad \forall v, s \in \mathbb{R}
$$

Hence, from the above inequality, we have

$$
\left(a_{0}-\sum_{i=1}^{m} c_{i}\right) \gamma \omega u_{1}(s) \leq \omega\left\|h_{1}\right\|_{C}
$$

Consequently, we have

$$
\left\|u_{1}\right\|_{C} \leq \frac{\left\|h_{1}\right\|_{C}}{\left(a_{0}-\sum_{i=1}^{m} c_{i}\right) \gamma} \triangleq \bar{R}
$$

Let $R>\max \{\bar{R}, r\}$. Then all the conditions of Lemma 2.9 are satisfied. By Lemma 2.9, we have

$$
i\left(Q, \Xi \cap \partial \Omega_{R}, \Xi\right)=1 .
$$

Combining (3.9) with (3.10) and by utilizing the additivity of fixed point index, we have

$$
i\left(Q, \Xi \cap \Omega_{r, R}, \Xi\right)=i\left(Q, \Xi \cap \Omega_{R}, \Xi\right)-i\left(Q, \Xi \cap \Omega_{r}, \Xi\right)=1 \neq 0 .
$$

Hence, $Q$ has at least one fixed point in $\Xi \cap \Omega_{r, R}$, which is the positive $\omega$-periodic solution of Eq. (1.1).

Remark 3 If we choose

$$
f\left(t, u\left(t-\tau_{1}\right), \ldots, u\left(t-\tau_{m}\right)\right)=\sum_{i=1}^{m} \frac{1}{i} u^{\frac{1}{2}}\left(t-\tau_{i}\right), \quad \forall u \in K_{C},
$$

we can prove that $(H 3)$ and $(H 4)$ hold. Hence, conditions $(H 3)$ and $(H 4)$ allow $f\left(t, x_{1}, \ldots, x_{m}\right)$ to be sublinear growth on $x_{1}, \ldots, x_{m}$.

\subsection{Conclusion}

In the present work, we establish some sufficient conditions on nonlinear term $f$ to guarantee the existence of positive $\omega$-periodic solutions of Eq. (1.1) in abstract Banach spaces. By using perturbation methods, we first prove the existence of positive $\omega$-periodic solutions of the linear problem corresponding to Eq. (1.1). Then the strong positivity estimation of the operator $T$ is established. The existence of positive $\omega$-periodic solutions of Eq. (1.1) is proved by utilizing fixed point index in cones. 


\section{Acknowledgements}

The authors are grateful to the editor and the reviewers for their constructive comments and suggestions for the improvement of the paper.

\section{Funding}

The research is supported by the National Natural Science Function of China (No. 11701457) and the Fund of College of Science, Gansu Agricultural University (No. GAU-XKJS-2018-142).

\section{Availability of data and materials}

Not applicable.

\section{Competing interests}

None of the authors have any competing interests in the manuscript.

\section{Authors' contributions}

All authors contributed equally in writing this paper. All authors read and approved the final manuscript.

\section{Publisher's Note}

Springer Nature remains neutral with regard to jurisdictional claims in published maps and institutional affiliations.

Received: 7 October 2019 Accepted: 18 March 2020 Published online: 16 April 2020

\section{References}

1. Chu, J.F., Fan, N., Torres, P.: Periodic solutions for second order singular damped differential equations. J. Math. Anal. Appl. 388, 665-675 (2012)

2. Chu, J.F., Zhou, Z.C.: Positive solution for singular nonlinear third-order periodic boundary value problems. Nonlinear Anal. 64, 1528-1542 (2006)

3. Deimling, K.: Nonlinear Functional Analysis. Springer, New York (1985)

4. Feng, Y.Q.: On the existence and multiplicity of positive periodic solutions of a nonlinear third-order equation. Appl. Math. Lett. 22, 1220-1224 (2009)

5. Graef, J., Kong, L.J., Wang, H.Y.: Existence, multiplicity and dependence on a parameter for a periodic boundary value problem. J. Differ. Equ. 245, 1185-1197 (2008)

6. Heinz, H.: On the behaviour of measure of noncompactness with respect to differential and integration of vector-valued functions. Nonlinear Anal. 7, 1351-1371 (1983)

7. Li, Y.X.: Positive solutions of higher-order periodic boundary value problems. Comput. Math. Appl. 48, 153-161 (2004)

8. Li, Y.X.: Positive periodic solutions of second-order differential equations with delays. Abstr. Appl. Anal. 2012, Article ID 829783 (2012)

9. Liu, X.Y., Li, Y.X.: Positive solutions for Neumann boundary value problems of second-order impulsive differential equations in Banach spaces. Abstr. Appl. Anal. 2012, Article ID 401923 (2012)

10. Wu, J., Wang, Z.C.: Two periodic solutions of second-order neutral functional differential equations. J. Math. Anal. Appl. 329, 677-689 (2007)

11. Wu, Y.X.: Existence, nonexistence and multiplicity of periodic solutions for a kind of functional differential equation with parameter. Nonlinear Anal. 70, 433-443 (2009)

12. Yan, S.H., Wu, X.P., Tang, C.L.: Multiple periodic solutions for second-order discrete Hamiltonian systems. Appl. Math. Comput. 234, 142-149 (2014)

13. Yang, H., Agarwal, R., Liang, Y.: Controllability for a class of integro-differential evolution equations involving non-local initial conditions. Int. J. Control 90, 2567-2574 (2017)

\section{Submit your manuscript to a SpringerOpen ${ }^{\circ}$ journal and benefit from:}

- Convenient online submission

- Rigorous peer review

- Open access: articles freely available online

- High visibility within the field

- Retaining the copyright to your article

Submit your next manuscript at $\boldsymbol{~ s p r i n g e r o p e n . c o m ~}$ 